\title{
FACIAL EXPRESSION CLASSIFICATION BASED ON PSEUDO ZERNIKE MOMENT INVARIANT, ZERNIKE MOMENT INVARIANT, PRINCIPAL COMPONENT ANALYSIS AND RADIAL BASIS FUNCTION NEURAL NETWORK
}

\author{
TRAN BINH LONG, TRAN HANH \\ Department of Computer Science, Lac Hong University
}

\begin{abstract}
This paper presents a new approach to classify facial expressions from frontal pose images. In our method, first Pseudo Zernike Moment Invariant (PZMI), Zernike Moment Invariant (ZMI) and Principal Component Analysis (PCA) are used to extract features from the global information of the images and then Radial Basis Function (RBF) Network is employed to classify the facial expressions based on the features which had been already extracted by PZMI, ZMI, PCA. Also, the images are preprocessed to enhance their gray-level, which helps to increase the accuracy of classification. For JAFFE facial expression database, the achieved rate of classification in our experiment is $98.76 \%$. This result shows that the proposed method can ensure a high accuracy rate of classification.
\end{abstract}

Tóm tắt. Phân lớp biểu lộ cảm xúc khuôn mặt là một bài toán quan trọng đã và đang được nhiều nhà nghiên cứu trong và ngoài nước quan tâm. Phân lớp chính xác biểu lộ cảm xúc khuôn mặt có thể ứng dụng trong nhiều lĩnh vực thực tế khác nhau: tâm lý học, thần kinh học. . .

Bài báo đề xuất một mô hình phân lớp biểu lộ cám xúc mới dựa trên việc kết hợp ba kỹ thuật rút trích đặc trưng (Pseudo Zernike Moment Invariant (PZMI), Zernike Moment Invariant (ZMI), Principal Component Analysis (PCA)) với mạng Radial Basis Function Neural (RBFN)); mô hình này tạm gọi là mô hình PZMI-ZMI-PCA-RBFN.

Việc thử nghiệm đánh giá mô hình so với một số phương pháp truyền thống khác trên cơ sở dữ liệu Jaffe cho thấy tính khả thi của kỹ thuật đề xuất.

Keywords. Facial expression classification, Pseudo Zernike Moment Invariant, Zernike Moment Invariant, Principal Component Analysis, RBF neural network.

\section{INTRODUCTION}

Facial expressions deliver rich information about human emotions, and thus play an essential role in human communications. For facial expression classification, data from static images or video sequences are used. In fact, there have been many approaches for facial expression classification using static images and image sequences $[1,2]$. These approaches first track the dynamic movement of facial features and then classify the facial feature movements into six expressions (i.e., smile, surprise, anger, fear, sadness, and disgust). Classifying facial expression from static images is more difficult than from video sequences because less information during the expression is available [3]. 
In order to design a highly-accurate classification system, the choice of feature extractor is very important. There are two approaches for feature extraction extensively used in conventional techniques [4]. The first approach is based on extracting structural facial features that are local structure of face images, for example, the shapes of the eyes, nose and mouth. This structure- based approach deals with local information. The second approach is based on statistical information about the features extracted from the whole image, so it uses global information [5].

Different structures for combining classifying systems can be grouped in three configurations $[6,7]$. In the first group, the classifying systems are connected in cascade to create pipeline structure. In the second group, the classifying systems are used in parallel and their outputs are combined to form parallel structure. Finally, the pipeline and parallel structures are combined to build up the hybrid structure. In this paper, we propose a human face expression classification system whose design is based on hybrid structure classifying system which enables evolutionary classification results by developing the features and selecting them for the classification problem.

Our proposed facial expression classification system is composed of four stages. In the first stage, the location of face in arbitrary images are detected. To ensure a robust, accurate feature extraction distinguishing between face and non-face region in an image, exact location of the face region is needed. We use a ZM-ANN technique in [8] for face localization and create a subimage which contains information necessary for classification algorithm. By using a sub-image, data irrelevant to facial portion are disregarded. In the second stage, different features are extracted in parallel structure from the derived sub-image. These features are obtained from the different domains. Three different feature domains are used for extracting features from input images. They are Pseudo Zernike Moment Invariant (PZMI), Zernike Moment Invariant (ZMI) and also Principal Component Analysis (PCA) [9]. In the third stage, classification was carried out by RBF neural network, based on the chosen features. This was done for each feature domain in parallel (Fig.1). In the last stage, the outputs of each neural network classifiers are combined to construct the identification. In this paper, the median method is selected for decision strategy and only automatic classification of facial expressions from still images in Japanese Females Facial Expression (JAFFE) database (Fig.2) [10] is discussed.

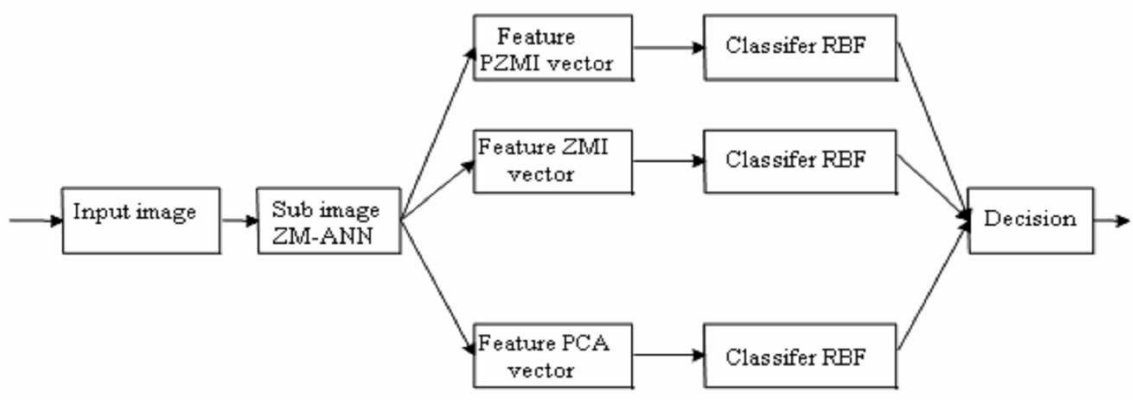

Fig. 1. The chart of PZMI-ZMI-PCA-RBF-GA system

The remainder of the paper is organized as follows. Section 2 describes the preprocessing procedure to get the pure expression image. Section 3 presents the feature domains. Section 
4 discusses the classification based on RBF network. Section 5 presents the experiments on the JAFFE facial expression database.

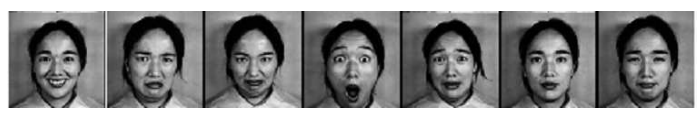

Fig.2. Examples of seven principal facial expressions in JAFFE: smile, disgust, anger, surprise, fear, neutral, and sadness (from left to right)

\section{PROPOSED FACE LOCALIZATION METHOD}

Many algorithms have been proposed for face localization and detection [11]. Face localization helps to find an object in an image to be used as a face candidate. The shape of this object resembles that of a face. Thus, faces are characterized by elliptical shape. In other words, an ellipse can approximate the shape of a face. A ZM-ANN technique presented in [8] provides the ability to find the best-fit ellipse to enclose the facial region of the human face in a frontal pose image.

The operation of face detection is done in two phases:

- In the first phase, representative Zernike vector is extracted from a selected image by a proper algorithm.

- In the second phase, a three - layer perceptron neural network, beforehand trained, receives on its input layer the Zernike moments vector and then gives on its output layer a set of points representing the probable contour of the face contained in the original image.

The neural network is used to extract statistical information contained in the Zernike moments and in the interactions closely related to the determined face region of the selected image. (Fig.3)

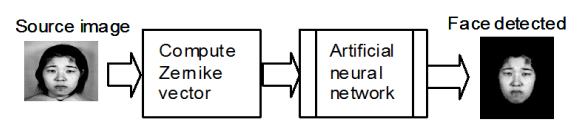

Fig.3. General diagram of the system detection

In general, the implementation of our method can be briefly described as follow:

- Computing vectors of Zernike moments for all the images $(N)$ in the work database.

- Constructing training database by randomly choosing $M$ images from the work database $(M<<N)$ and identifying Zernike moment vectors $Z_{i}$ corresponding to $M$ images.

- Manually delaminating face area in each image of the training database based on a set of points representing the contour $C_{i}$ of each treated face. The points include the top, bottom, left and right of identified image, all of which form an ellipse whose semi-major axis $a=45$, semi-minor axis $b=40$, rate $b / a \approx 8 / 9$ (see Fig.4.a).

- Training neural network on the set of $M$ couples $\left(Z_{i}, C_{i}\right)$.

The test and measurement of the performance of the network obtained after training operation are done on $(N-M)$ the other images in the work database. 

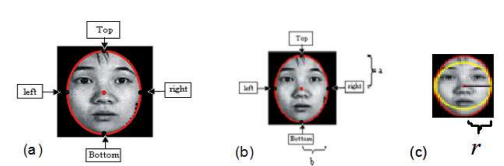

Fig.4. Center of ellipse, circle determined by basing on the top, bottom, left and right

\section{FEATURE DOMAINS}

In order to design a good face expression classification system, the choice of feature extractor is very crucial. To design a system with less complexity, the feature vectors should contain the most pertinent information about the face to be classified. In the proposed system, different feature domains are extracted from the derived sub-images in parallel structure. Therefore, this approach can extract more characteristics of face images for classification purpose and three different kinds of feature domains have been selected. They are PZMI, ZMI and PCA.

\subsection{Zernike moment invariant}

Zernike moments, initially introduced by F.Zernike [12], form part of the general theory of the geometrical moments. Unlike general geometrical moments, Zernike moments are built on a set of orthogonal polynomials. These polynomials are the basic elements of the construction of an orthogonal base given by

$$
V_{n m}(x, y)=R_{n m}(x, y) \exp \left(j m \tan ^{-1}\left(\frac{y}{x}\right)\right),
$$

where $x^{2}+y^{2} \leq 1, n \geq 0,|m| \leq n$ and $n-|m|$ is even and radial polynomials $R_{n m}$ are defined as

$$
R_{n m}(x, y)=\sum_{S=0}^{(n-|m|) / 2} S_{n,|m|, S}\left(x^{2}+y^{2}\right)^{n-2 S}
$$

where,

$$
S_{n,|m|, S}=(-1)^{S} \frac{(n-S) !}{S !\left(\frac{n+|m|}{2}-S\right) !\left(\frac{n-|m|}{2}-S\right) !} .
$$

The complex Zernike moments of order $n$ and repetition $m$ are given by

$$
Z M_{n m}=\frac{n+1}{\pi} \sum_{x} \sum_{y} f(x, y) V_{n m}^{*}(x, y)
$$

\subsection{Pseudo zernike moment invariant}

PZM is similar to $Z M$ except that the radial polynomial is defined as

$$
R_{n m}(x, y)=\sum_{S=0}^{(n-|m|)} \frac{(-1)^{S}(2 n+1-S) !\left(x^{2}+y^{2}\right)^{n-S}}{S !(n-|m|-S) !(n+|m|+1-S) !}
$$

where $n=0,1,2, \infty$, and $m$ is an integer subject to constraint $|m| \leq n$ only. 


\subsection{Principal component analysis}

PCA is a well-known statistical technique for feature extraction. Each $M \times N$ image in the training set is a row concatenated to form $M N \times 1$ vectors $\widetilde{A}_{l}$ Given a set of $N_{T}$ training images $\left\{\widetilde{A}_{l}\right\}_{i=0,1,2, \ldots, N_{T}}$ the mean vector of the training set is obtained as

$$
\bar{A}=\frac{1}{N_{T}} \sum_{i=1}^{N_{T}} \widetilde{A}_{l} .
$$

The average vector are subtracted out from the training vectors as

$$
A_{i}=\widetilde{A}-\bar{A}, i=1,2,3, \ldots, N_{T} .
$$

An $N_{T} \times M N$ matrix $A$ is constructed with the $A_{i}^{T}$ as its row vectors. The singular value decomposition of $A$ can be written as

$$
V^{T} A U=\left|\sum\right| 0 \mid,
$$

where $\Sigma$ is an $N_{T} \times N_{T}$ diagonal matrix with singular values $s_{i}>0$ arranged in descending order, $V$ and $U$ are $N_{T} \times N_{T}$ and $M N \times M N$ orthogonal matrices, respectively. $V$ is composed of the eigenvectors of $A A^{T}$, while $U$ is composed of the eigenvectors of $A A^{T}$. These are related by

$$
\hat{U}=A^{T} U
$$

where $\hat{U}$ consists of the eigenvectors of $A A^{T}$ corresponding to the non-zero singular values. This relation allows to solve a smaller $N_{T} \times N_{T}$ eigenvalue problem for $A A^{T}$, and to subsequently obtain $\hat{U}$ by matrix multiplication.

The projection of a face vector onto the space of $N_{T}$ eigenfaces results in an $N_{T}$ - dimensional feature vector of projection weights. As PCA has the property of packing the greatest energy into the least number of principal components, the smaller principal components which are less than a threshold can be discarded with minimal loss in representational capability. This dimensionality reduction results in face weight vectors of dimensions $\widetilde{N}_{T}<N_{T}$. An appropriate value of $\widetilde{N}_{T}$ can be chosen by considering the Basis Restriction Error (BRE) as a function of $\widetilde{N}_{T}$ [9]. This gradual decrease in error is significant for recognition techniques based on eigenfaces where storage and computational performance are directly related to $N_{T}$.

\subsection{Simulations}

\subsubsection{Zernike/Pseudo Zernike Moments}

In this section, we test the performances of ZM/PZM and hope that the information contained in a moment is rich and distinctive. The image representation ability can be estimated by reconstructing the image using moments with a particular order. Fig.5 shows the images reconstructed from the two kinds of moments with orders ranging from $10,12, \ldots 24$.

It is well known from the figure that the PZM performs better than ZM. In practice, when the orders of ZM/PZM exceed a certain value, the quality of the reconstructed image degrades 
quickly. This is due to the numerical instability problem inherent with ZM/PZM. From this point of view, we chose the order 35 with 36 feature vector elements for ZM, and order 20 with 41 feature elements for PZM in our experiment.

(a)

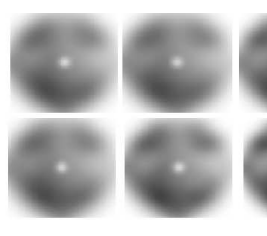

Fig.5. Image reconstruction: (a). ZM. (b).PZM

\subsection{2. $P C A$}

Let a face image $I(x, y)$ be a two-dimensional $N$ by $N$ array of (8-bit) intensity values. An image can be considered as a vector of dimension $N^{2}$, so that a typical image of size 256 by 256 becomes a vector of dimension 65.536, or, equivalently, a point in 65.536-dimensional space. An ensemble of image, maps to a collection of points in this huge space.

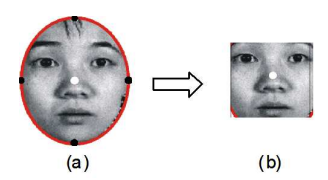

Fig.6. (a) image $80 \times 80$, (b) Feature space $30 \times 30$

For reduce number of dimensions. The face images can be represented in the way by projecting the data in the image space onto the face space (also called Eigenfaces) as described in the previous section. The dimension of the projected data in the feature space is much smaller than that in the original image space. After the Eigenfaces are obtained, we may transform face image into feature space by a simple projection operation. The projections constitute a vector $V=\left[v_{1}, v_{2}, \ldots, v_{k}\right]$ called Eigen-vectors, where $k$ is the dimension. In practice, $k$-dimensional equivalently 30 with images preprocessed (size $80 \times 80$ ). Fig.6.

Then the eigenvectors of the correlation matrix of the training data are computed. Next, the principal components of the projection of each image are collected as the training set for RBFN. For a test image, we project it onto the Eigen-space obtained previously. Then, the projection vector is fed into RBFN.

\section{CLASSIFIER DESIGN}

In this paper, an RBF neural network $[13,14]$ is used as a classifier in a face expression classification system, where the inputs to the neural network are feature vectors derived from the proposed feature extraction technique described in the previous section.

\subsection{RBF neural network description}

Neural network technology offers a number of tools such as learning and adaptation, generalization and robustness, feature extraction and distributed representation. The neural network approach has been fruitfully shown in solving face classification problems. The radial 
basis function neural network (RBFN) theoretically provides such a sufficiently large network structure that any continuous function can be approximated within an arbitrary degree of accuracy by suitably choosing radial basis function centers [15]. The RBFN is trained by using sample data to approximate a function in multidimensional space. A basic topology of RBFN is depicted in Fig.7. The RBFN is a three-layered network. The first layer constitutes input layer in which the number of nodes is equal to the dimension of input vector. In the hidden layer, the input vector is transformed by radial basis function by the following activation function

$$
\varphi\left(\bar{x} ; \bar{c}_{j}\right)=\exp \left(-\frac{1}{2 \sigma^{2}}\left\|\bar{x}-\bar{c}_{j}\right\|^{2}\right)
$$

where $\left\|\bar{x}-\bar{c}_{j}\right\|$ denotes a norm- (usually Euclidean distance) of the input data sample vector $\bar{x}$ and the center $\bar{c}_{j}$ of radial basis function. The $k$ th output is computed by the following equation

$$
F_{k}(x)=\sum_{j=1}^{m} w_{k j} \varphi\left(\bar{x} ; \bar{c}_{j}\right),
$$

where $w_{k j}$ represents a weight synapse associated with the $j$ th hidden unit and the $k$ th output unit with $m$ hidden units.

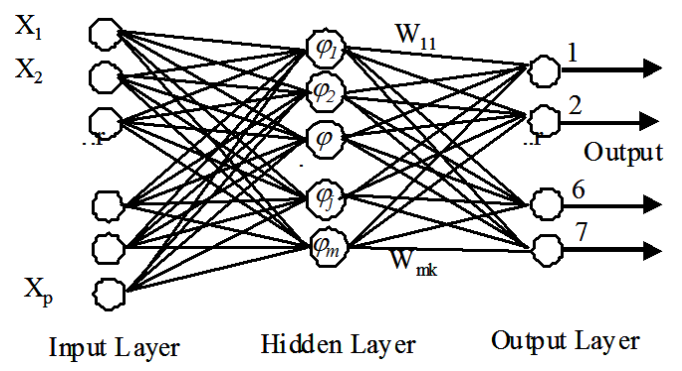

Fig.7. Basic topology of RBFN

\subsection{RBF neural network classifier design}

We employed the RBFN to classify the facial expressions images in the Eigen-space domain extracted via ZMI, PZMI, PCA as described in the previous section. The architecture is depicted in Fig.8. The major advantages of RBFN over other models such as feed-forward neural network and backpropagation are its fast training speed and local feature convergence.

To design a classifier based on RBF neural networks, in the input layer of the neural network, we set an amount of input nodes which are as many as the number of feature vector elements. The number of nodes in the output layer is 7 , which is equivalent to 7 facial expressions of image classes. Initially, the RBF units are equal to the number of output nodes, and these RBF units increase if the classes are overlapped.

\subsection{The fundamental algorithms of the system}

We construct two primary algorithms of the application. The first algorithm is used to extract features from the derived sub-images in parallel structure (ZM,PZM,PCA). The second 
algorithm is used to train RBF neural networks and the midean rule for classifying.

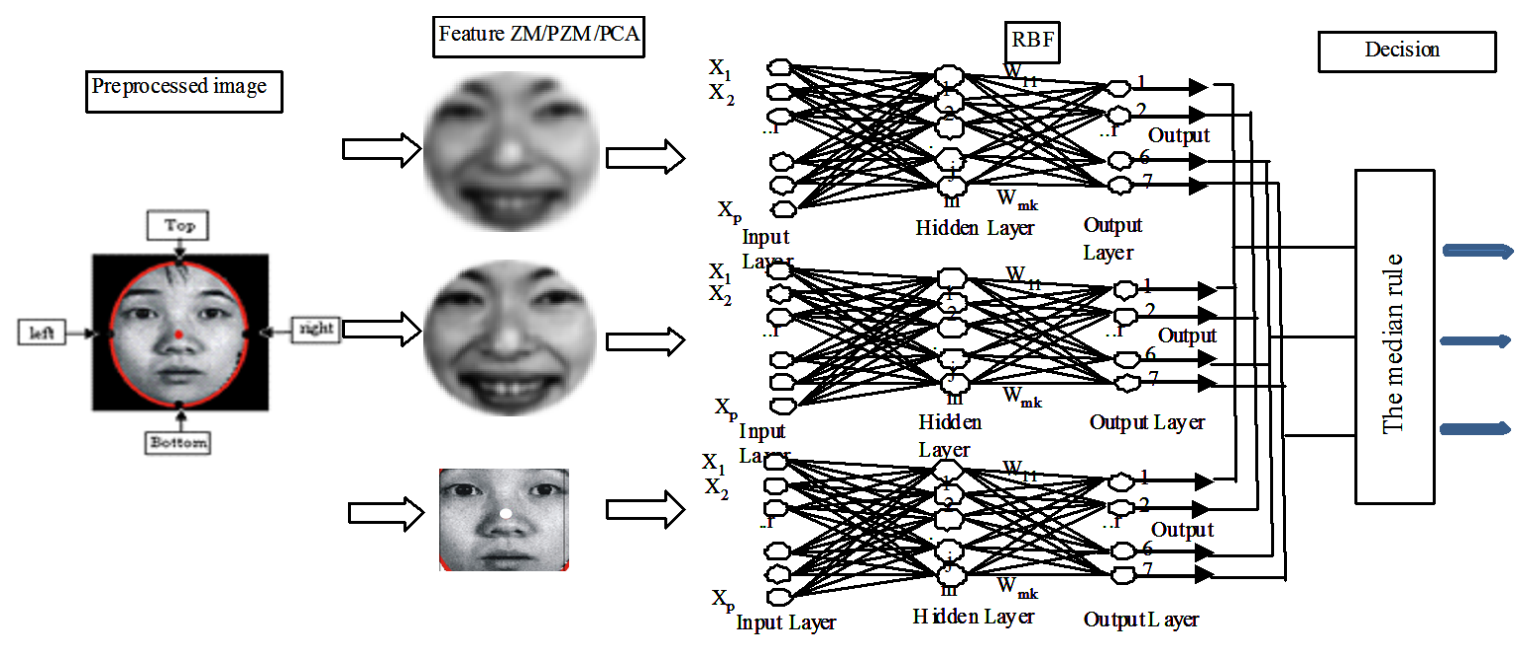

Fig. 8. ZM,PZM,PCA,RBFGA system

\section{Extracting feature algorithm}

Step 1. Extracting feature of image use ZM. Creating feature vector with order 35.

Step 2. Extracting feature of image use PZM. Creating feature vector with order 20.

Step 3. Extracting feature of image use PCA. Creating feature vector with k-dimensional equivalently 30 .

\section{Object classifying algorithm}

Step 1. Each feature vector is input of a RBF neural network.

Neural network 1: Feature vector elements of ZM equal to 36 corresponds 36 input nodes of input layer. The number of RBF units of hidden layer equals to the number of nodes output of output layer equal to 7 corresponds 7 facial expression.

Neural network 2: Feature vector elements of PZM equal to 41 corresponds 41 input nodes of input layer. The number of RBF units of hidden layer equals to the number of nodes output of output layer equal to 7 corresponds 7 facial expression.

Neural network 3: Feature vector elements of PCA equal to 30 corresponds 30 input nodes of input layer. The number of RBF units of hidden layer equals to the number of nodes output of output layer equal to 7 corresponds 7 facial expression.

Step 2. With each network, use weight the numbers(evaluating the reliability of each individual network). In this framework, the most commonly used the median rule can be obtained by using median of sum of weighted numbers. This method represent the most important scheme that combines individual classifiers achieving better recognition rates. Then the object is decided to belong to class.

\section{EXPERIMENTAL RESULTS}

In this section, we demonstrate the capabilities of the proposed PZM-ZM-PCA-RBFN approach in classifying seven facial expressions. A sample of the proposed system with three 
different feature domains and the RBF neural network have been developed. In this example, for the PZM and ZM, moments order 20 and 35 have been considered as feature vector elements. The feature vectors for these domains have 41 elements for the PZM and 36 elements for the ZMI. Also for the PCA feature vector, 30 largest PCA number from each image have been created [16]. The proposed method is evaluated in terms of its classification performance using the JAFFE female facial expression database [10], which includes 213 facial expression images corresponding to 10 Japanese females. Every person posed 3 or 4 examples of each of the seven facial expressions (happiness, sadness, surprise, anger, disgust, fear, neural). Two facial expression images of each expression of each subject are randomly selected as training samples, while the remaining samples without overlapping are used as test data. We have 140 training images and 73 testing images for each trial. To investigate the local effect of the source images, we use images with the size $80 \times 80$. Since the size of the JAFFE database is limited, we perform the trial over 3 times to get the average classification rate. Our obtained classification rate is $98.76 \%$ (Table 1 ).

Table 1. Classification rate (\%) of the proposed pzmi-zmi-pca-rbf model

\begin{tabular}{|l|l|l|l|l|l|l|l|}
\hline Test & Sadness & Smile & Disgust & Neutral & Surprise & Fear & Anger \\
\hline 1 & 99.68 & 98.58 & 98.95 & 98.01 & 98.68 & 97.88 & 98.45 \\
\hline 2 & 98.88 & 98.88 & 99.76 & 98.87 & 99.64 & 99.46 & 98.95 \\
\hline 3 & 98.52 & 97.85 & 98.92 & 97.85 & 98.84 & 98.45 & 98.86 \\
\hline
\end{tabular}

To compare the effectiveness of the proposed method with the Single Feature Neural Network (SFNN) human face expression classification systems, we have developed the SFNN systems using the PZMI+RBF[18], ZMI+RBF [17] and PCA+RBF[19]. For these systems, we have selected the PZMI as feature domains with order 9 and 10 which have 40 elements; the ZMI with order 9 and 10 which include 21 feature elements and finally the PCA with 30 largest values. The comparison PZMI-ZMI-PCA with each individual classifiers as a function of class number is shown in Table 2. It can be seen from the obtained results that the classification rate of the PZMI-ZMI-PCA is much better than that of any other individual classifiers and that although the output of each individual classifier may agree or conflict with each other, the PZMI-ZMI-PCA search for a maximum degree of agreement among the conflicting supports of the face pattern.

Table 2. Comparative results of the classification rate (\%) of sfnn approaches

\begin{tabular}{|l|l|}
\hline Methods & Rate \\
\hline ZMI+RBF & $97.77 \%$ \\
\hline PZMI+RBF & $98.33 \%$ \\
\hline PCA+RBF & $85.68 \%$ \\
\hline Proposed method & $98.76 \%$ \\
\hline
\end{tabular}

For the classification performance evaluation, a False Acceptance Rate (FAR) and a False Rejection Rate (FRR) test are performed. These two measurements yield another performance measure, namely Total Success Rate (TSR)

$$
T S R=\left(1-\frac{\mathrm{FAR}+\mathrm{FRR}}{\text { tatal number of accesses }}\right) \times 100 \% \text {. }
$$


The system performance can be evaluated by using Equal Error Rate (EER), where $\mathrm{FAR}=\mathrm{FRR}$. A threshold value is obtained based on Equal Error Rate criteria where FAR $=\mathrm{FRR}$. Threshold value of 0.2954 is gained for PZM-ZMI-PCA as measure of dissimilarity.

Table 3 shows the testing result of verification rate with order moments from setting 20 (moments order 20) for PZMI-ZMI and (30) PCA based on their defined threshold value.

The results show that the application of PZMI-ZMI-PCA as feature extractors can best perform the classification.

Table 3. Testing result of verification rate of pzmi-zmi-pca

\begin{tabular}{|l|l|l|l|l|}
\hline moment & thres & FAR(\%) & FRR(\%) & TSR(\%) \\
\hline PZMI-ZMI-PCA & 0.2954 & 1.7998 & 2.1674 & 98.76 \\
\hline
\end{tabular}

We have compared our proposed method with some of the existing facial expression classification techniques on the same Jaffe database. This comparative study indicates the usefulness and the utility of the proposed technique.

The three other methods taken for the comparison are HRBFN+PCA [22], Gabor + PCA +LDA [20], GWT+DCT+RBF [21] (see in Table 4).

Table 4. comparative results of the classification rate (\%) of different approaches

\begin{tabular}{|l|l|}
\hline Methods & Rate \\
\hline Gabor + PCA+LDA [16] & $97.33 \%$ \\
\hline GWT+DCT+RBF [17] & $89.11 \%$ \\
\hline HRBFN+PCA [10] & $95.68 \%$ \\
\hline Proposed method & $98.76 \%$ \\
\hline
\end{tabular}

\section{CONCLUSIONS}

The performance of orthogonal Pseudo Zernike Moment Invariant (PZMI), Zernike Moment Invariant (ZMI), Principal Component Analysis (PCA) and Radial Basis Function Neural Network (RBFN) in the facial expression classification system have been presented in this paper. The combination of orthogonal moments at high orders, PCA and RBF neural network contain more information about face image and improve the classification rate. The highest classification rate of $98.76 \%, F A R=1.7998 \%$ and FRR $=2.1674 \%$ with Jaffe database are achieved using our algorithm, which represents the overall performance of this facial expression classification system. The proposed algorithms, PZMI+ZMI+PCA+RBF, possess some advantages: orthogonality and geometrical invariance. Therefore, they are able to minimize information redundancy as well as to increase the discrimination power.

\section{REFERENCES}

[1] I. A. Essa, A. P. Pentland, Coding, analysis, interpretation, and recognition of facial expression, IEEE Trans. Pattern Analysis and Machine Intelligence 19 (1997) 757-763.

[2] B. Fasel, J. Luettin, Automatic facial expression analysis: a survey, Pattern Recognition 36(2003) 259-275.

[3] X. W. Chen and T. Huang, Facial expression recognition: a clustering-based approach, Pattern Recognition Letter 24 (2003) 1295-1302. 
[4] J. Daugman, Face Detection: A Survey, Computer Vision and Image Understanding 83 (3) (2001) 236-274.

[5] L. F. Chen, H. M. Liao, J. Lin and C. Han, Why recognition in a statistic-based face recognition system should be based on the pure face portion: a probabilistic decision-based proof, Pattern Recognition 34 (7)(2001) 1393-1403.

[6] T.K.Ho, J.J.Hull and S.N.Srihari, Decision combination in multiple classifier systems, IEEE Trans. On Patt. Anal. and Mach. Intel. 16 (1) (1994) 66-75.

[7] Y. Lu, Knowledge integrations in a multiple classifier system, Applied Intelligence 6 (2) (1996) $75-86$.

[8] DangThanhHai, LeHoangThai, LeHoaiBac, "Facial boundary detect in images using Moment Zernike and Artificail Neural Network", Dalat Universitys Information Technology Conference, $2010(39-49)$.

[9] M. Truk and A. Pentland, Eigenfaces for recognition, Journal Cognitive Neuroscience 3 (1) (1991) 71-86.

[10] www.kasrl.org/jaffe.html.

[11] J. Daugman, Face detection: A survey, Computer Vision and Image Understanding 83 (3) (2001) 236-274.

[12] F. Zernike, Physica 1934.

[13] J. Haddadnia, M. Ahmadi, and K. Faez, A hybrid learning RBF neural network for human face recognition with pseudo Zernike moment invariant, IEEE International Joint Conference On Neural Network (IJCNN 02), Honolulu, Hawaii, USA, May 2002 (11-16).

[14] L. Yingwei, N. Sundararajan, and P. Saratchandran, Performance evaluation of a sequential minimal radial basis function (RBF) neural network learning algorithm, IEEE Transactions on Neural Networks 9 (2) (1998) 308-318.

[15] S. Haykin, Neural Networks: A Comprehensive Foundation, Macmillan College Publishing Company, New York, 1994.

[16] Javad Haddadnia, Majid Ahmadi, Karim Faez, $N$-feature neural network human face recognition, Image and Vision Computing 22 (2004) 1071-1082.

[17] TranBinhLong, LeHoangThai, TranHanh, Facial expression classification using zernike moment invariant and artificial neural network, Processing of the Second IITA International Joint Conference on Artificial Intelligence (IITA-JCAI), Shanghai, China, 2010 (221-224).

[18] Tran Binh Long, Le Hoang Thai, Tran Hanh, Fecial expression classification method based on pseudo zernike moment and radial basis function network, Proceeding of IEEE $3^{\text {rd }}$ Int. Conf. Machine Learning and Computing (ICMLC), Singapore, February 26-28, 2011 (310-313).

[19] Thai Hoang Le, Nguyen Thai Do Nguyen, Hai Son Tran, Facial expression classification using principal component analysis and artificial neural network, Proceeding of IEEE $3^{\text {rd }}$ Int. Conf. Machine Learning and Computing (ICMLC), Singapore, February 26-28, 2011 (306-309). 
[20] Hong-Bo Deng, Lian-Wen Jin, Li-Xin Zhen, Jian-Cheng Huang, A new facial expression recognition method based on local gabor filter bank and pca plus lda, International Journal of Information Technology 11 (11) (2005).

[21] Praseeda Lekshmi.V, Dr.M.Sasikumar, A neural network based facial expression analysis using gabor wavelets, World Academy of Science, Engineering and Technology 42 (2008).

[22] Daw-Tung Lin, Facial expression classification using pca and hierarchical radial basis function network, Journal of Information Science and Engineering 22 (2006) 1033-1046.

Received on January 08 - 2011

Revised on October 27 - 2011 